\title{
UNA EVALUACIÓN DE LA VITALIDAD LINGÜÍSTICA DEL INGLÉS CRIOLLO DE LIMÓN: SU VIGENCIA O SU DESPLAZAMIENTO ${ }^{1}$
}

\author{
Anita Herzfeld
}

\begin{abstract}
RESUMEN
La historia nos ha mostrado que siempre que exista una situación de lenguas en contacto, se desarrolla o un bilingüismo armonioso o una todopoderosa colonización. También todos saben que la relación entre lengua e identidad puede resultar en consecuencias divergentes en tales circunstancias, ya sea una asimilación cultural o una fusión o la extinción de una lengua. Este trabajo enfocará el caso de una minoría de hablantes del criollo limonense (CL), basado en el inglés, en contacto con el español (E) de Costa Rica. Se verá que los hablantes del CL frecuentemente alternan el criollo limonense con el español (CL-E), y al mismo tiempo importan préstamos y hacen sustituciones incorporando al español en el CL. Aunque es riesgoso predecir el futuro de la relación entre ambas lenguas, esta fusión -más que la extinción del criollo limonense- puede prevalecer si sus hablantes consideran que su lengua es un elemento importante de su identidad.

Palabras clave: lenguas en contacto, criollo limonense, extinción, fusión, alternancia de códigos.
\end{abstract}

\begin{abstract}
History has shown that whenever there is a situation of languages in contact, it develops either into a harmonious bilingualism or an overpowering colonialism. It is also well known that the relationship between language and identity may bring about divergent consequences in such circumstances; notably, cultural assimilation or the fusion or the extinction of a language. This paper will focus on the case of a minority of English-based Limonese Creole (LC) speakers in contact with Spanish-speaking (S) Costa Ricans. It will be apparent that LC speakers frequently make use of LC-S code-switching and imported loans and substitutions thus incorporating Spanish into Limonese Creole. Even though it is risky to predict the future of that relationship, this fusion -rather than the extinction of the Creole- may prevail if its speakers consider their language an important element of their identity. Key words: contact languages, Limonese Creole, extinction, fusion, code-switching.
\end{abstract}

\section{Introducción}

Una minoría Afro-Caribeña de aproximadamente 55000 personas habla el criollo limonense basado en el inglés. El país es predominantemente caucásico y hablante del español. Los hablantes del criollo han vivido en la provincia de Limón, por más de 200 años. La

\footnotetext{
Dra. Anita Herzfeld. Profesora Titular. Centro de Estudios Latinoamericanos, Universidad de Kansas. Correo electrónico: herzfeld@ku.edu
}

Recepción: 12- 03- 2012

Aceptación: 18- 03- 2012 
provincia es marcadamente diferente del resto del país en lo que se refiere a su geografía, historia, población, economía, lengua y cultura.

A pesar de esta marcada individualidad, los costarricenses en general se enorgullecen de ser una población física y culturalmente homogénea, descendiente de los colonizadores españoles. Recientemente, los antropólogos y otros cientistas sociales han tratado de concientizar al público acerca de su origen mestizo y su ancestro africano (Bozzoli 1993: 2) ${ }^{2}$. Sin embargo, sólo en círculos académicos se conocen cabalmente las variedades de lenguas habladas en Costa Rica, tanto las indígenas como el criollo limonense $(\mathrm{CL})^{3}$. La mayoría de la gente da por sobrentendido el cambio "necesario" de las lenguas indígenas y el criollo al español (E), como si fuera un hecho que no precisa ser cuestionado como parte de la identidad nacional.

Este trabajo va a tratar la vigencia o el desplazamiento de la relación lengua-identidad entre los afro-limonenses que están inmersos en un medio hispánico. En lo que sigue, voy a presentar al criollo limonense y el marco socio-histórico en el que existe. Luego, el trabajo enfoca el remplazo del acrolecto del CL por el E como resultado de la alternancia de códigos así como también por el uso de importaciones morfológicas y sustituciones como resultado de préstamos. Es importante anotar que al estudiar un criollo, la lengua se considera generalmente un continuo lingüístico, que se extiende desde el basilecto al acrolecto, con un mesolecto entre ambos extremos. Mientras que el basilecto es la forma más radical del criollo y el acrolecto es la que está más cerca de la lengua relexificadora (el inglés, en este caso), el mesolecto comparte formas de ambos extremos. Todos los hablantes se mueven a lo largo de este continuo, usando formas de estos lectos que están determinadas por una serie de factores. Después de la clasificación de los datos micro-sociolingüísticos recolectados en grabaciones de hablantes del CL quienes navegan el continuo, el trabajo termina con comentarios especulativos sobre el futuro del criollo limonense, basados en una taxonomía de factores definidos por Giles, Bourhis, and Taylor (de ahora en adelante GBT 1977: 308), que pueden ayudar a determinar la vitalidad de una lengua ${ }^{5}$.

\section{Una breve descripción del marco socio-histórico del criollo limonense}

Los esclavos africanos que arribaron al Valle Central de Costa Rica en el siglo XVI se convirtieron eventualmente en miembros de la sociedad indígena y española de la época por mestizaje, y sus lenguas se perdieron. Los afro-caribeños que llegaron en la onda migratoria de la segunda mitad del siglo XIX a Costa Rica vinieron en su mayoría desde Jamaica para trabajar en la construcción del ferrocarril que uniría a San José, la capital, con Puerto Limón en la costa Caribeña (Apéndice No. 1). Nunca pudieron regresar a su tierra; en cambio, permanecieron en Costa Rica para trabajar en el puerto o en las plantaciones de banana, ambas propiedades de la United Fruit Company en esa época. Es sólo ahora, más de un siglo más tarde, después de que se construyó una carretera que une a la capital con la costa Caribeña, y como resultado de la emigración hispana a la costa de gente que buscaba trabajo, que los matrimonios mixtos han llegado a ser muy comunes.

El idioma de los afro-limonenses, que yo he llamado 'criollo limonense', viene del criollo jamaiquino, la lengua materna de la mayoría de los inmigrantes afro-caribeños. Se conoce popularmente como /mekaytelyuw/, que viene del jamaiquino 'Make a tell you something' (inglés estándar (IE) 'Let me tell you something'). La mayoría de los hablantes del CL vive en la provincia de Limón, aunque últimamente muchos han emigrado al Valle 
Central y han adoptado el español, la lengua oficial del país. Esto ha afectado al criollo en forma significativa. Sin embargo, a pesar de las campañas educativas para homogeneizar a los hablantes, la lealtad de esta minoría hacia su etnicidad está presente aún hoy en muchas formas: en el uso de su lengua, en su comida, en sus juegos y deportes y en su música y canciones. Vale la pena preguntarse cuánto tiempo más esta situación de lenguas en contacto se mantendrá vigorosamente.

Hay varios factores socio-lingüísticos que han contribuido a que el E afectara al CL como resultado de su situación de contacto: 1) el E se ha convertido en la lengua de prestigio en Limón en los últimos cincuenta años como consecuencia de decisivas campañas educativas; 2) fuertes sentimientos nacionalistas han surgido en la región; 3) un cambio radical ha tenido lugar en la demografía, y 4) las comunicaciones entre Puerto Limón y el Valle Central se han incrementado notablemente, ya que ahora la costa está a sólo dos horas y media de la capital. Lo que esto significa, en cuanto a los hablantes del LC se refiere, es que actualmente, ellos están inmersos en el proceso de restructurar sus reglas lingüísticas, incorporando más y más formas de la lengua de prestigio (el E) en su gramática.

En el panorama bosquejado más arriba, la situación de lenguas en contacto entre el CL y el E es asimétrica tanto en lo social como en términos socio-psicológicos. Por eso, podemos asumir con certeza que el CL está sufriendo una influencia mayor del E, es decir de la lengua socialmente dominante de la mayoría, que viceversa. Sin embargo, a pesar de la restructuración que está ocurriendo en este momento, el CL es aún una lengua viva, aunque desde la década de 1980 muchas (pero de ninguna manera todas) las familias criollo-limonenses han abandonado la socialización básica de sus hijos en la lengua de su patrimonio ${ }^{4}$. En este momento, los niños, quienes antes tenían la oportunidad de asistir a clases de inglés estándar -ya sea privadas o auspiciadas por las iglesias, impartidas en su mayoría por maestros jamaiquinos que también hablaban y enseñaban el IE a partir del CL- tienen hoy acceso muy limitado a esas clases. Además, aunque muchos de esos niños todavía poseen un conocimiento activo del CL, sus clases en las escuelas públicas, que antes estaban constituidas en su mayoría por niños afrolimonenses, hoy constan, en su mayoría, de hablantes del E.

Los jóvenes limonenses hablantes del criollo usan ambos el E y el CL con muchos préstamos del E para tratar temas que tienen que ver con la escuela o "college" (colegio, high school), especialmente cuando la conversación trata de las materias que ellos estudian, y aún más si los temas requieren el uso de vocabulario técnico. Por otra parte, es muy probable que si los diálogos tienen que ver con política y negocios, especialmente los temas relacionados con la realidad nacional de Costa Rica, serán expresados en E, o en el CL con muchos préstamos del E.

Las conversaciones sobre el trabajo de la oficina, y la terminología legal y oficial, las noticias, el servicio público, son más "fáciles" de tratar en E, especialmente para la gente joven. La mayoría de las jóvenes afro-limonenses prefieren que sus enamorados (i.e. significant others) les hablen en E, porque piensan que es más "dulce” y más romántico. El CL se usa, en cambio mucho más para contar bromas y para intercambios que significan pasarlo bien (como en "vacilar entre amigos", es decir para "bromear," o "tomarle el pelo a alguien"); eso también ocurre cuando los hablantes sostienen 'peleas' verbales, y cuando usan "malas palabras". En breve, se prefiere el CL como código lingüístico cuando lo que se habla en un grupo íntimo está vinculado con los sentimientos de identidad con la gente del "grupo de uno". Por ahora, la solidaridad de un grupo y el sentido de identidad de los miembros de un grupo étnico se mantienen en el CL. Sin embargo, si uno trata de impresionar a alguien con su nivel educativo 
y sus conocimientos técnicos, es muy probable que ese diálogo se exprese en E, ya que eso significaría un estatus de superioridad técnica, poder y cierta sofisticación intelectual obtenida en la cultura hispánica, y no en la comunidad nativa.

En este trabajo, se tratará de evaluar la "nueva" vitalidad lingüística del CL, la lengua subordinada, como resultado de la imposición de elementos de la lengua "super-ordinaria": el E. Giles, Bourhis and Taylor (1977: 308) definen "vitalidad" de un grupo etno-lingüístico como "eso que hace que un grupo probablemente se conduzca como una entidad colectiva distintiva y activa en situaciones de inter-grupo". Se deduce de esto, que las minorías etno-lingüísticas cesan de existir si no tienen vitalidad de grupo, $\mathrm{y}$, al revés, un grupo lingüístico tiene más probabilidades de sobrevivir si tiene gran vitalidad, es decir que en lugar de funcionar como individuos aislados, los miembros de un grupo se dirigen uno al otro en situaciones de inter-grupo. Teniendo en cuenta entonces que las consideraciones extra-lingüísticas, tales como ciertas realidades políticas, históricas y económicas también juegan un papel significativo en la manutención o desplazamiento de una lengua, los autores Giles, Bourhis y Taylor (1977: 308-317) proponen un modelo en el cual ciertas variables estructurales determinan la vitalidad de los grupos etnolinguíísticos. Ellos presentan tres categorías de factores, los cuales son el estatus, la demografía, y el apoyo institucional; más específicamente son los siguientes:

a. Factores de estatus: Cuatro son los factores que se consideran en este rubro:

- Estatus económico: Citando a GBT (1977: 310), este corresponde al "grado de control que ha obtenido un grupo que habla cierta lengua sobre la vida económica de esa nación, región o comunidad".

- Estatus social: Este se refiere al "grado de estima que un grupo lingüístico tiene para sí mismo.” (GBT 1977). Mientras que una baja auto-estima bien puede debilitar su visión de ellos mismos y su lengua, una auto-estima alta bien puede elevarla.

- Estatus socio-histórico: Como lo mencionan GBT (1997: 310): "sin tener en consideración el resultado de estas [luchas], los ejemplos históricos se pueden usar como símbolos de movilización".

- Estatus de la lengua: El prestigio y el respeto que una lengua ejerce en una comunidad, ayuda a determinar su mantenimiento.

b. Factores demográficos: GBT (1997: 312) cita ciertas variables como contribuyentes a la vitalidad de grupos etno-lingüísticos, entre otras, ellos consideran al territorio nacional, una concentración y proporción de grupo.

- Los factores de distribución demográfica: Si los miembros de una comunidad de habla consiguen mantener cohesión en su arreglo espacial, es muy probable que ellos puedan mantener su idioma.

- Los factores de número de grupos: Los números absolutos, los índices de nacimientos, los matrimonios mixtos, la inmigración y la emigración son también indicativos de las chances de sobrevivir que tiene la lengua de una minoría.

c. Factores de apoyo institucional: Estos tienen que ver con "el grado de apoyo formal e informal que un idioma recibe de las instituciones de una nación, región, o comunidad" (GBT 1997: 315). Un desglose más detallado de estas variables, expandido por Miller (2000) figura en el Apéndice No. 2. 


\section{El remplazo del acrolecto por una segunda lengua}

Cuando dos o más lenguas están en contacto cercano, como es el caso de los hablantes del CL con el E en Costa Rica, se produce una alternancia de códigos. Por alternancia de códigos, entiendo aquí la acción lingüística adoptada por hablantes bi/multilingües, quienes cambian de una lengua a otra(s) en interacciones conversacionales. Este fenómeno de alternancia, es decir el ir de una lengua a la(s) otra(s), ya sea en la mitad de una oración usando palabras, frases u oraciones de la(s) otra(s) lengua(s), o contestando en la otra lengua de hablantes bi/multilingües, es muy complejo; implica un total conocimiento de las gramáticas usadas y puede señalar una serie de significados distintivos, como será aparente más abajo (Foley 1998: 333). En todo caso, es un importante recurso comunicativo en muchas comunidades bi- y multilingües (Gumperz 1982). Les permite a los hablantes manipular todas sus lenguas simultáneamente, $y$, cuando se practica frecuentemente, contribuye a incorporar préstamos de elementos de una lengua a otra. Como tal, un fenómeno común es la transferencia de préstamos de una lengua $\mathrm{A}$, como sustitución morfológica de diferente tipo a una lengua $\mathrm{B}$, ya sea en forma totalmente integrada o, por el contrario, adaptando esos préstamos a la otra lengua. Estos procesos ocurren usualmente en situaciones de contacto, cuando la lengua de la mayoría socialmente dominante, aumenta su influencia sobre la lengua de la minoría. Como consecuencia, la lengua dominante afecta a la lengua socialmente no-dominante mucho más que vice-versa (Rindler Schjerve 1998: 221), y eventualmente, hasta puede llevar a un cambio o desplazamiento de una lengua por la otra, es decir puede contribuir a la muerte de una lengua (Dorian 1989).

En el presente estudio de caso, es difícil predecir cuáles son las implicaciones de estos procesos en el futuro del CL, ya que a pesar de la pérdida de ámbitos lingüísticos por el dominio que tiene el E en el país, la lengua minoritaria no puede considerarse de ninguna manera obsoleta. Spence (1993: 194) estudió varios ámbitos lingüísticos tales como logias, juegos de dominó, partidos de beisbol, la novena noche (ninth night), el mercado, la escuela, el barrio, los centros de salud y la iglesia, que habían sido tradicionalmente ámbitos del CL, y determinó que en sólo dos o tres de ellos todavía prevalece el CL vigorosamente.

\section{El estudio}

\subsection{Los datos y consideraciones metodológicas}

El corpus que provee los ejemplos de las varias categorías de alternancia de códigos así como las sustituciones morfológicas fue obtenido durante varios viajes de campo de diferente duración a la provincia de Limón durante un período de 30 años, a partir del año 1973. Se hicieron más de 500 horas de grabaciones de 280 hablantes seleccionados al azar. Las muestras del CL se obtuvieron en forma sistemática como respuestas a un cuestionario preparado y memorizado anteriormente, que indujeron a una conversación guiada. Ese cuestionario fue administrado por mi asistente, el señor Owen Hammond, hablante del CL, quien con sus modales amistosos y sus inteligentes intervenciones logró ganar fácil acceso a los hablantes del CL y los hizo sentirse cómodos durante las grabaciones. (Véase Herzfeld 2002 para una detallada descripción de la metodología empleada para obtener los datos en una forma estadísticamente valedera). 
Los temas variaban de acuerdo con los intereses de los hablantes entrevistados, de manera que a los pescadores se les preguntaba si alguna vez habían estado en peligro de perder la vida en alta mar, para que contaran sus experiencias en esas circunstancias; a las amas de casa se les pedían recetas, que ellas estaban encantadas de compartir con Owen; a los profesionales se les preguntaba frecuentemente acerca de los problemas políticos locales, nacionales e internacionales; a los estudiantes de escuelas secundarias se los hacía contar anécdotas sobre sus profesores favoritos o más temidos. Los ejemplos de alternancia de códigos como también las sustituciones morfológicas fueron luego extraídos de los datos obtenidos; estos ejemplos fueron clasificados para mostrar la inestabilidad lingüística del criollo, que actualmente está pasando por grandes cambios debido a la presencia notoria de la lengua dominante en comunidades de habla mixta.

Como se mencionara anteriormente, el propósito principal de este trabajo es el de llevar a cabo un análisis estructural del curso de relaciones intergrupales y sus efectos en la vitalidad etno-lingüística de una comunidad de habla del CL. Además de las consideraciones anteriores, se puede argüir que una evaluación objetiva hecha por el grupo de hablantes sobre la vitalidad de su idioma puede ser tan importante como la realidad objetiva que se describe más abajo.

\subsection{Las preguntas de la investigación}

La situación lingüística actual de Limón puede describirse como un desplazamiento, abierto y gradual, cuya dinámica específica está caracterizada por un uso intensivo de alternancia de códigos y modelos de préstamos, como se ejemplifica más abajo. Sin embargo, para observar más sistemáticamente el fenómeno de alternancia de códigos así como los préstamos y las sustituciones como expresiones de una asimetría e inestabilidad sociolingüística subyacente -que podría poner seriamente en peligro la existencia del CL para llevarlo a un desplazamiento de la lengua- se tendrán en cuenta las tres categorías de variables (estatus, demografía, y el apoyo institucional) en el análisis preliminar de los datos finales.

Dado el panorama descrito aquí, el objetivo de este trabajo es por lo tanto proveer el contexto necesario para comprender el proceso lingüístico del desplazamiento de una lengua. ¿Será que el fenómeno de alternancia de códigos y la importación de préstamos -que son el resultado de una situación despareja del diferente prestigio de las lenguas en contacto- inducen al desplazamiento de una de las lenguas? ¿Será que los factores extra-lingüísticos de naturaleza socio-políticos contribuyen a responder a esta pregunta? Y aún más importante, ¿Será que el CL soportará la presión del E, la lengua oficial de la mayoría de la población, o está destinado a desaparecer en las próximas generaciones? ¿Existe un factor en particular que podría inclinar los acontecimientos hacia una forma u otra?

\subsection{Una clasificación de los ejemplos de alternancia de códigos}

Los subtítulos que aparecen más abajo son parte de un esfuerzo preliminar por clasificar los ejemplos extraídos de los datos obtenidos, en categorías posibles de alternancia de códigos. En realidad, las circunstancias en que ocurrieron pueden ser mucho más sutiles que lo reflejado en los subtítulos. Por otra parte, se prestan para discutir la objetividad de la vitalidad etno-lingüística de la sociedad del CL, usando estas variables como determinantes del gran impacto sufrido por el mantenimiento o defunción de la lengua minoritaria. 


\subsection{Cambio debido a circunstancias socio-culturales de las situaciones de habla}

a. Cambio de interlocutor

b. Cambio de tema

c. Principio o fin de una conversación

d. Falta de competencia en el idioma

e. El estímulo de un préstamo

f. Una cita directa

g. Las madres que llaman la atención de sus niños

Algunos ejemplos de estas categorías se presentan a continuación.

\subsubsection{Cambio de interlocutor}

Si dos jóvenes están hablando en el CL y un joven hispano se les acerca, todos pasan a hablar en español inmediatamente. Lo opuesto también ocurre; dos jóvenes, hablantes del CL y del $\mathrm{E}$, que conversan en $\mathrm{E}$, al agregarse al grupo una tercera persona de cierta edad hablante del CL, hace que la conversación se vuelva al CL inmediatamente.

En un diálogo reproducido aquí, obtenido durante el trabajo de campo llevado a cabo en Puerto Limón, la abreviatura CL sirve para indicar Criollo Limonense, IE para Inglés Estándar; E, para Español; EE es para denotar Español Estándar y las iniciales que siguen corresponden a los nombres de los interlocutores, protegiendo así su identidad y su privacidad "variacionista." Como el CL no es una lengua escrita, se ha adoptado aquí un sistema fonémico simplificado para facilitar la mecanografía del proceso de las transcripciones y la lectura de los textos.

(1) CL O -wish languij yu taak wid fren dem?

IE O - -What language do you speak with friends?'

CL Y - wid fren/ wel som a dem yu av spanish an inglish

IE Y - 'With friends, well some of them you have (talk in) Spanish and English'.

CL Y -yu taak wid di spanish gerl dem/ dwon andestan inglish

IE Y - You talk with the Spanish girls, (they) don't understand English'.

CL Y - so yu av to taak wid dem spanish/ an wid di blak gerl dem inglish

IE Y -'So you have to talk Spanish with them, and with the black girls, English'.

CL Y -somtaym a taak wid dem spanish/ bikoz now di mwo a di blak piypel dem,

IE Y - 'Sometimes I talk with them (in) Spanish, because most of the black people,'

CL Y - dem taak mor spanish/ mor dan inglish...

IE Y - speak more Spanish, more than English...

\subsubsection{Cambio de tópico}

El clasificar a los tópicos de conversaciones con sus correspondientes lenguas es un tanto artificial. Un hablante bilingüe elige una u otra lengua para expresarse, de acuerdo con su identificación con el sistema de valores de esa cultura en particular. Además, las relaciones 
interpersonales así como las presiones sociales desempeñan un papel muy importante en la selección de la lengua respecto del tema a tratarse.

El ejemplo que sigue menciona los cambios que sufre el CL; los hablantes usan el CL para el intercambio de ideas y además agregan el uso de la alternancia de códigos:

(2) CL BA - evriy yer wiy gowin taak mor rifayn inglish/ antil wiy riych trap rayt intu di amerikan inglish...

EI BA -Every year we (are) going (to) talk more refined English until we reach (the) trap right into (the) American English...

CL FH -wel/ ay down biliyv so/ yuw nuwo way/ bikozin op to now/yuw av e greyt distant.../fers tin dat emmm wiy staat tu mor miks spanish wid inglish...

EI FH -well, I don't believe so, you know why? Because up to now, you have a great distance...First thing that $\mathrm{mmm}$ we start to mix more Spanish with English...

Si hablaran de problemas matemáticos, seguramente lo harían en EE.

\subsubsection{Al principio o al fin de una conversación}

Los diálogos que tiene la gente en la calle revelan el uso del E al principio o al final de un intercambio casual entre muy buenos conocidos. El ejemplo que sigue ilustra el uso del E al principio de un intercambio entre amigos.

(3) E CB - ¿kwándo regresaste de inglatera?

E CB $-¿$ Cuándo regresaste de Inglaterra?

CL KH - ay neva gow inglan/ a gow tu olan

IE $\mathrm{KH}$-I didn't go to England, I went to Holland.

4.3.1.4. Falta de fluidez en uno de los idiomas

Un hablante bilingüe que no se siente cómodo en ambas lenguas va a tratar de conversar en la lengua que habla mejor.

(4) CL FH - a gowen tel yu di plyen trut/ yu faget/ riyliy/ ay siy personaliy/ siy se wel

IE FH -'I am going to tell you the plain truth, you forget, really, I saw (it) personally, (I) saw well'.

CL FH -iz not posibel dat dem faget dem languij/ bot dem faget.

IE FH -'(It) is not possible that they (should) forget their language, but they forget'.

IE AH -haw kam?

IE AH -'How come?'

EE FH -bueno, talbes no te lo podría eksplikar en inglés/ así no...

IE FH (Well, perhaps I could not explain it to you in English, this way no...)

EE FH - Bueno, tal vez no te lo podría explicar en inglés, así no...' 
CL AH -way not? yuw kyaan sey in inglish aal dat yu wan?

IE AH - 'Why not? You cannot say in English all that you want?'

EE FH -no kreo ke se lo pueda eksplikar en inglés...

EE FH - 'No creo que se lo pueda explicar en inglés...'

IE FH (I don't think I can explain it to you in English)

\subsubsection{El estímulo de un préstamo}

En algunas ocasiones, después de un préstamo en E, el hablante continúa hablando en ese idioma:

(5) CL M - shiy onliy rayt layk se "tú"/ an layk so / entiende no?

IE M - She only writes like say, "tú", and like so, entiende no?

EE M - "Ella sólo escribe digamos, "tú”, y así, entiende, no?’

IE M - 'She only writes, like say, "tú” and like so, you understand, don't you?'

4.3.1.6. Para introducir una cita directamente

(6) CL CF - ... baal im/ im no nwo wa apin/ at nayn oklok yu aftu gow tu bed an sliyp

IE CF - '... Bawl him, he did not know what (had) happened, at nine o'clock, you have to go to bed and sleep'.

CL CF - aal dem raatid no gud/ man/ as yuw ir nayn oklok/ dem kom an aks yu now

IE CF - 'All (of) them (said in wroth) not good, man, as you hear nine o'clock, they come and ask you now'.

CL CF -layk se/ yu stan op de now an ay iz de gobermen/ ay kom op an se

IE CF - 'like say, you stand up there now and I am the government, I come up and say'.

CL CF —“oiga tu sédula"/yuw se l“no"/an yuw se bot.../“no tengo"/im se "bueno"

IE CF - "Oiga, tu cédula", you say "no" and you say but... "No tengo", he says, "bueno"

IE CF —“Listen, your I.D." You say, "no" and you say but..." I don’t have one", he says, "o.k.")

CL CF — "pele gayo" / yu nwo wa dat miyn?

IE CF - "Pele gallo", you know what that means?'

4.3.1.7. Las madres que llaman la atención a sus niños

(7) CL O -ow moch childrin yu av?

IE O - 'How many children do you have?'

CL S - siks

IE S - 'Six' 
CL O -ow moch yer dem av?

IE O - 'How old are they?'

CL S -siy di biyebiy dyer?/dirigiéndose al bebé):jkayáte bos!/ i de debil wanself...

IE S - 'See the baby there (addressing the baby): Calláte vos! (Be quiet!) He is the devil himself...'

(8) CL O -wish languij yu riyd mwor?

IE O - '(In) which language you read more?'

CL S -lay riyd bwot/ (dirigiéndose al bebé que está llorando) ¡káyese!/

IE S - 'I read both (addressing the baby who is crying) Cállese! (Be quiet!)'

\subsubsection{Cambio como dispositivo retórico}

a. Énfasis al repetir una observación en dos lenguas

b. Énfasis de una oración que el oyente puede no esperar

c. Énfasis por contraste

d. Para hacer una observación parentética

e. Para palabras o temas tabú

f. Inseguridad de un bilingüe imperfecto

Algunos ejemplos de estas categorías se presentan a continuación.

4.3.2.1. Énfasis al repetir una observación en dos lenguas

(9) CL IF - dem kyan gow into de likel salita/ intu de ?aal

IE IF - 'They can go into the little salita (hall), into the hall'.

(10) CL R -tu aks di aplifment of progris/layk sel luxo/ av tinz dat yu wan in yu hows

IE R - To ask for the "upliftment" of progress, like say, lujo (luxury) to have things that you want in your house'

CL R -layk se lagzeri...

IE R -'Like say, luxury...'

4.3.2.2. Énfasis de una oración que sorprende al oyente

En este ejemplo la joven (IF), que responde a la pregunta que le hiciera el joven (OH), se sorprende por el interés tan personal que él tiene en su vida privada amorosa.

(11) $\mathrm{CL} \mathrm{OH} \mathrm{-im} \mathrm{av} \mathrm{entrada?}$

IE OH - 'He has entrada?'

CL IF - no/ not entrada/ bika may fada not lie tu gii permishon/...de por sí

IE IF - No, not entrada (permission to come into the house), because my father is not here to give (him) permission, de por sí...(in reality)' 
CL IF - ay down niyd may fada permishon...

IE IF - 'I don't need my father's permission...'

\subsubsection{3. Énfasis por contraste}

En el ejemplo que sigue, la joven (IF) le explica a su interlocutor que ella se las arregla para darle órdenes a su novio, aunque ninguna otra chica antes que ella había podido hacerlo.

(12) CL IF -im tel mi kozin se dat no gerl eva hangel im/ not hangel im yet

IE IF - 'He told my cousin, say that no girl has ever "handled" him, not "handled" him yet'

CL IF - bot ay kyaan manda im an tel im enitin ay fiyl an enitin ay wan

IE IF - 'But I can manda (boss) him and tell him anything I feel and anything I want'.

\subsubsection{Para hacer una observación parentética}

Los "hedges" se usan frecuentemente en E, y en el CL se usa "Es que.." o "Se = Say" muy parecido al uso de "like" en inglés.

(13) $\mathrm{CL} \mathrm{OH} \mathrm{-u} \mathrm{iz} \mathrm{yu} \mathrm{bwayfren?}$

IE $\mathrm{OH}-$-Who is your boyfriend?'

CL IF - es ke... ay kyaan tel yuw/ fa yuw mayt nwo im...

IE IF -'Es que...I cannot tell you, for you might know him...'

(14) CL CF —lisin bway/ may basilon wen ay smaal iz ful erown fling shot

IE CF - 'Listen boy, my vacilón when I was small was to fool around fling-shots'.

CL CF -likin down em bord/ bika diai/...yu nwo...

IE CF - 'striking down the birds, because de ahí...tú sabes'.

\subsubsection{Para palabras o temas tabús}

En este ejemplo, el hablante se siente avergonzado de que le pusieran una multa por mal comportamiento.

(15) CL SS -bika dem gowin multa mi...

IE SS - 'Because they are going to multa me...'

4.3.2.6. Inseguridad de un bilingüe imperfecto

(16) CL UB -jehovas witnis/ testigo de xeoba...

IE UB -'Jehova's Witness, testigo de xeoba...'

(17) CL RP - shiy av tuw taykel/ tuw diploma/ ¿no?

IE RP - 'She has two titles (degrees), two diploma ¿no?' 


\subsection{Préstamos y substituciones}

Además del remplazo en el acrolecto debido a situaciones socio-culturales y cambios producidos por el uso de dispositivos retóricos (todos ellos interferencias inducidas por el cambio), hay otros cambios que se originan por préstamos. Por préstamos (i.e. borrowings) entiendo aquí la incorporación que un hablante nativo hace en su lengua materna, de las características de otro idioma. El punto importante de esta definición (Thomason 2001: 250) es que estos préstamos no son producidos por el aprendizaje imperfecto de un hablante bilingüe.

En lo que sigue, se presentan varios casos de préstamos del E. He distinguido entre los que el CL adopta totalmente (integración del E en el CL), y aquellos que son elementos que participan en el continuo proceso de difusión (interferencia per se).

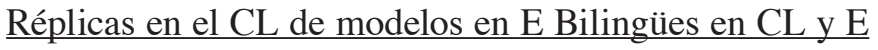 \\ Integración --> Adoptado por toda la comunidad de habla \\ Interferencia --> Adoptado sólo por ciertos hablantes bilingües}

\section{Préstamos importados}

a. Integración

b. Interferencia

II. Préstamos adaptados (o ajustados)

a. Formación inversa

b. Formación de nombres (sustantivos)

c. Participio Presente

d. Adición del sufijo $\{-n e s s\}$

Más abajo se ejemplifica esta clasificación y los préstamos aparecen en negrita. Como en los ejemplos mencionados anteriormente, las iniciales que preceden a cada parlamento del diálogo corresponden a los nombres de los hablantes que fueron grabados en el campo.

\subsubsection{Préstamos importados}

\subsubsection{Integración}

En el tipo de préstamos importados tipificados como integración "se introduce en la lengua un nuevo morfema que adopta la forma fonémica que muestra una identificación diafónica con un morfema de la lengua origen" (Haugen 1956: 52). Los miembros de la comunidad de habla usan palabras en E, pero ellas funcionan como si fueran palabras en el CL. La integración tiene lugar especialmente entre los hablantes jóvenes, quienes se expresan más cerca del mesolecto del continuo. Por ejemplo:

(18) $\mathrm{CL} \mathrm{O}-$ a shu yuw av plentiy tu tel miy...

IE I am sure (that) you have plenty to tell me...'

CL I -layk wat?

IE I 'Like what?' 
CL O -layk wat yuw duw/ we yuw liv/ de barrio we yuw liv...

IE O 'Like what you do, where you live, the barrio (district, neighborhood) where you live...'

(19) CL O -im av entrada?

IE $O$ 'Does he have entrada (permission to come in)?'

CL I - no, not entrada/ bika may fada

IE I 'No, not entrada (permission to come in) because my father'

CL I -not /ie tu gii permishon...de por síl

IE I 'is not here to give permission...de por sí (in any case)'

CL I -ay dwon niyd may fada permishon...

IE I 'I don't need my father's permission'

(20) CL O -so dat miyn yu not gowin marid suwn?

IE O 'so that means you are not going to marry soon?'

CL I - $\quad$ in se so? Ay se falta plentiy...

IE I 'who says so? I say falta (it will take) a long time...'

\subsubsection{Interferencia}

Hay varios tipos de préstamos que pueden considerarse "interferencias", porque no han sido totalmente adoptados por todos los hablantes del CL.

a. Estos son ejemplos que muestran palabras en E que vienen primero a la mente, cuando se habla el CL:

(21) CL L - -yu fada kyatch yuw/ put yuw in kana fi tan op

IE L 'your father catches you, puts you in a corner to stand up'

CL L - castigar yuw...

IE L 'castigar (chastises, punishes) you...'

(22) CL I _ - seben yer egow now/ ay dwon bay now regalo

IE I '(It is) seven years now since I haven't bought a regalo (present)

LC I -fa mi fada...

IS I 'for my father...'

(23) CL G -bihayn de man dat staatid de bronka

IE G 'behind the man that started the bronca (row)'

(24) CL I - dem kyan gow intu de likl salita...

IE I 'they can go into the little salita (hall)...' 
b. Este grupo de ejemplos ilustran el caso definido de la siguiente manera, "Ningún morfema nuevo es introducido, pero el lexema que es un préstamo está traducido por la redistribución de morfemas que ya se encuentran en la lengua" (Haugen 1956: 52). Estas son palabras inglesas que o bien han cambiado, o han extendido o limitado de alguna manera su significado, bajo la influencia de formas similares en E ("cognados falsos"). Por ejemplo,

(25) CL I - di wonli taym ay siy yu/ yu profesal

IE I - 'The only time I saw you, you (were) an instructor'.

CL I - an ay kudn showt tu yuw...

IE I - 'and I couldn't "shout” at you' (say hello)'

CL O -no/ bot yu siy mi aftawor...

IE O - No, but you saw me afterwards...'

CL I -no / ay neba siy yu/ jes tudye/ wen mis anita prizent wi (EE nos presentó)

IE I - 'No, I haven't seen you, just today, when Ms. Anita introduced us'

(26) CL O -wa yu gowin stodiy wen yu kom owt kolij (EE colegio)

IE O - 'What are you going to study when you come out (from) college?' (high school)

(27) CL FP - kownt mi a stworiy / EE cuéntame)

IE FP —'Tell me a story...'

(28) CL CG - de siy haz riyliy retrosiydid... (E retrocedido)

IE CG — 'The sea has really regressed' (the tide has ebbed)

c. En los ejemplos que siguen, los modelos sintácticos y las expresiones idiomáticas aparecen "traducidos" en el CL, como si fueran a adoptar una forma similar a la del EE. Por ejemplo,

(29) CL I - if yu stodiy fi padre/ O / a tel yu se

IE I - - If you study for padre (the priesthood) O, I tell you that,'

CL I -lpyur/ onli pyur gerl wil gow church...

IE I -'(Nothing but= EE puras) girls will go (to) church...'

(30) CL I -de milk kot op (se cortó)

IE I - 'The milk curdled'

(31) CL - ay keym bak to limon an ay tuk nayt kolij (IE college=EE colegio)

IE 'I came back to Limón and I went to night school,'

CL -an tuk owt may bachiyerato... (EE y saqué)

IE - - and (I) obtained my bachelor's...' 
(32) CL - ay wan tu mek a shwo... (hacer un show)

IE - - I want to produce a show...'

\subsubsection{Préstamos adaptados (o ajustados)}

Estos son préstamos del E que han sido adaptados a la morfología del CL, la lengua que se los apropia.

(33) Formación Inversa o Regresiva

E /konserbar/ "conservar": CL /konserbyet/

IE "conserve": IE *"conservate"

$\mathrm{E} /$ fomentar/ "fomentar": CL /fomentyet/

IE "foment": IE *"fomentate"

(34) Formación de Sustantivos

E /asignasión/ "asignación”: CL /asigneyshen/

IE "assignment"

E /manifestación/ "manifestación”: CL /manifesteyshon/

IE "demonstration"

(35) Formación del Participio Presente agregando -ing\} al verbo en E

E /konfesarse/ "confesarse": CL /konfesarin/

IE "confessing"

E /rechasar/ "rechazar": CL /rechasarin/

IE "rejecting"

(36) Formación de Sustantivos Abstractos agregando \{-ness\} a la base E

E /romántiko/: CL /romantiknis/

IE "romantic, romanticism"

Algunos de estos ejemplos aparecen ilustrados en las oraciones siguientes:

(37) $\mathrm{CL} \quad$-wi tray tu fomentyet it a likl mwo bay givin di smala

IE - "We try to foster it a little more by giving the smaller" (E fomentar)

LC - man a likel mo heyd...

IE - - 'man a little more aid...'

(38) LC -yu konfesarin now...

IE - - you are confessing now..." (E confesar)

(39) LC - - mi down now way/ bot we dem gii mi asignyeshen tu duw/

IE - 'I don't know why, but when they give me an assignment to do'

(40) LC -in di kolij..aal di taym fiyl liezy tu duw it...

IE - 'in the college (school)...all the time (I) feel lazy to do it'. 


\subsection{Consideraciones teóricas}

Como no existe una teoría comprensiva sobre la alternancia de códigos, el estudio de los datos de alternancia de códigos del CL-E, presentado originariamente en español en Herzfeld (2005: 331-355) desde el punto de vista sociolingüístico, siguió el modelo desarrollado por Myers-Scotton (1993). En ese modelo, se asume que la alternancia de códigos es un recurso de la comunicación bilingüe y que las formas observables de la alternancia de códigos están sujetas a restricciones sociolingüísticas y estructurales de la lengua específica en la que se dan los pares involucrados. Dados los ejemplos examinados, sigo aquí las directrices del modelo para determinar si se puede asumir que la situación del E-CL es un caso de desplazamiento de lenguas o no.

Para la conveniencia del lector, se incluye aquí un resumen del modelo de MyersScotton. Consiste de un mecanismo de préstamo profundo (deep borrowing) y otro de desplazamiento (shift). El primero consta a su vez de préstamos tanto de léxico como de ítems de gramática, ya que en las conversaciones en que tiene lugar la alternancia de códigos, una lengua es dominante, tanto en términos de la frecuencia de morfemas como en términos de ser la selección (o alternativa) esperada, y no marcada, para el discurso en consideración (Myers-Scotton 1993: 113-154). También distingue entre una lengua "matriz" (LM) y una lengua "enclavada" (LE) (o insertada) (1993: 66-74), que se activan en forma diferente. Con tal propósito, es importante diferenciar entre morfemas de contenido y de sistema, hablando en general, ítems de una clase abierta o de una clase cerrada (1993: 98-102). El concepto de lengua "matriz" se basa en dos consideraciones: a) la alternancia de códigos, ya sea que fuera no-marcada (i.e. la selección esperada) o marcada (i.e. la selección inesperada), es un fenómeno social, y por lo tanto se fundamenta en normas y valores sociales; b) la alternancia de códigos está sujeta a limitaciones estructurales a las que tanto la matriz como la lengua enclavada contribuyen en formas diferentes. Cuando los morfemas de ambas lenguas se combinan, es decir, que los constituyentes de la Lengua Matriz (LM) + la Lengua Enclavada (LE) (o insertada) se combinan en una alternancia intra-fraseal, el modelo predice que las formas de la LE tienen que acomodarse a los requisitos estructurales de la LM (i.e. la LM provee el marco morfo-sintáctico para los préstamos de la LE).

Estas consideraciones teóricas llevan a Myers-Scotton a desarrollar diferentes panoramas, dependiendo de la situación lingüística. En situaciones donde hay alternancia de códigos en que la lengua matriz se mantiene, como es el caso del CL, la interferencia de la lengua dominante (EE) tiene diferentes resultados de aquellos que producen desplazamiento o muerte. Estas van desde situaciones de contacto intensivo con manutención de la lengua y una condición de relativa no-marcación de ambos códigos, a través de una fase de relexificación, cambio de marcación, y por consiguiente, a un cambio de roles entre la LM y el EE, y finalmente a situaciones de la muerte de una lengua. La afirmación más notable de Myers-Scotton es que la presencia de la LE en alternancia de códigos intra-fraseal (LM+ constituyentes de la LE) va a aumentar, tan pronto como la manutención de la lengua se vuelva inestable, no importa que sea por un cambio en la competencia de los hablantes o a un cambio en las normas interactivas de ciertos tipos de discurso. En otras palabras, lo que ella predice es una primera fase de relexificación sin convergencia gramatical.

Más adelante, cuando el desplazamiento de la lengua se ha asentado, la lengua LE va aumentando el rol de LM, un estado de cosas que claramente facilita la importación de estructuras gramaticales en las alternancias expresadas. 
De acuerdo con Rindler Schjerve (1998: 224), los elementos importados no necesitan ser abruptos, pero pueden ser lentos y descontinuados dependiendo de las variadas competencias e identidades que definen a los grupos de hablantes y las identidades que definen a los grupos de hablantes en tales situaciones de desplazamiento de idiomas (monolingües activos que han cambiado su lengua por la LE, o bilingües semi-hablantes quienes están en proceso de cambiar, bilingües que mantienen su vieja lengua LM). El principio básico de estas situaciones es que la alternancia de códigos en situaciones de contacto inestable favorece los préstamos y sobre todo, préstamos profundos. Es a través de la alternancia que la lengua no-dominante cambia. Desde este punto de vista, el CL no está aún en el paso de desplazamiento de lengua, sino que está pasando por una fase de relexificación en el proceso que resulta de la alternancia de códigos.

\subsection{Las consideraciones de vitalidad}

Los datos que se han considerado en este trabajo son parte de un corpus mucho más grande (Herzfeld 2002) que no ha sido analizado aún de acuerdo con los principios de la metodología de la "vitalidad". Sin embargo, algunos comentarios cualitativos pueden ser apropiados. Siguiendo el orden mencionado anteriormente, Giles et al. (1977: 308-317), mencionan que entre los factores de estatus, el que tiene más importancia es el estatus económico. Partiendo del marco socio-histórico presentado en este trabajo, está claro que los hablantes del CL tienen poco control económico sobre su destino financiero. Viven en la provincia de Limón que provee entradas substanciales de capital al país como resultado de las actividades del puerto, turismo e importaciones, pero pocas de estas entradas permanecen en la provincia. En general, los hablantes del CL laboran en trabajos estacionales conectados a actividades portuarias y, mucho menos que en el pasado, en plantaciones de banana, estas han ido a parar hoy en manos de los inmigrantes nicaragüenses.

Hablando en términos generales, su estatus económico deja mucho que desear en comparación con el de los hablantes de la lengua de la mayoría, especialmente fuera de Limón. Dorian (1982: 47) ha señalado que "la lealtad hacia una lengua persiste mientras las circunstancias económicas y sociales son conducentes a esa lealtad, pero si alguna otra lengua resulta tener un valor más grande, comenzará un desplazamiento a esa lengua". Si se pensara en base a este criterio únicamente, está claro que la existencia del CL estaría en serio peligro de desaparecer. Las consideraciones económicas son muy importantes, pero hay casos en que resultan irrelevantes. Bien puede ser que un grupo obedezca fielmente los mandatos pragmáticos del momento; sin embargo, puede estar en contra de los imperativos económicos. Si un grupo siente que mantener su propia lengua no contribuye a mejoras materiales, pero al mismo tiempo no lleva a pérdidas materiales, es decir, que es lo suficientemente "seguro de sí mismo" como para conservar un capital cultural y tradicional, es posible que esa cultura active emociones y sentimientos que conserven la lengua (Edwards 1994: 117). Es decir que "las preocupaciones económicas y pragmáticas son centrales, pero tambíén puede ser que estén enterradas bajo muchas renovadas capas de cultura y tradición" (Edwards 1994: 118).

En cuanto al estatus social, este es un criterio muy complejo de establecer en la actualidad. Mientras que es verdad que por muchos años los hablantes del CL se han sentido discriminados y en consecuencia consideraron a su lengua como "inferior" (i.e. "broken English"), últimamente ha habido un resurgimiento de su afecto por la música de calipso y sus letras, siempre expresadas en el CL, así como el uso del CL en bromas, al jugar al dominó, en los eventos deportivos, y otros lugares domésticos. Así es que ahora parece haber una 
revaluación de lo que el CL significa para sus hablantes, en consecuencia el CL parece estar ganando la batalla que mantiene con el E.

Con respecto al estatus socio-histórico del CL, la historia de los hablantes del CL ha sido una de luchas por defender su existencia como entidad colectiva. Tomemos el ejemplo del señor C. King, un vecino activista, recientemente fallecido. Gracias a sus incansables esfuerzos y los de sus amigos han logrado mantener viva la United Negro Improvement Association (UNIA) en Puerto Limón, así como las logias que ellos establecieron para cooperar con la comunidad afro-costarricense. Además, se han preocupado por conseguir que en las escuelas se celebre el Día de los Afro-Costarricenses, así como el Día de las Culturas, la semana del 12 de octubre, y se siguen celebrando ambas festividades hasta el día de hoy. Yo creo que este es el caso de los hablantes del CL, por lo menos por ahora, ya que estos símbolos históricos parecen haber conducido a la solidaridad del grupo y por lo tanto han contribuido en alguna medida a su vitalidad.

En referencia al estatus de la lengua, es muy posible que la falta de estandarización escrita del criollo pueda ser considerada en parte la razón de la falta de prestigio del idioma para la comunidad. Pero las desventajas pueden ser canceladas por el orgullo de formar parte de un grupo exclusivo de hablantes que usan este código especial, particularmente en vista de la poesía que se ha publicado últimamente en el CL, así como la producción literaria a la que han contribuido distinguidos hombres y mujeres de letras de origen afro-costarricense, hablantes del CL, tales como Eulalia Bernard y Quince Duncan, entre muchos otros. Claramente, estas variables inclinan el balance de los factores de estatus a favor del mantenimiento del CL.

En cuanto a Giles et al. (1977), los factores demográficos, y más específicamente, los factores de la distribución del grupo, en realidad, prácticamente, la minoría hablante del CL se ha mantenido en su suelo nativo tradicional, aunque en los últimos veinte años, muchos de sus miembros jóvenes se han ido a la capital del país en busca de mejores oportunidades. Sin embargo, están visiblemente vinculados a sus ancestros ya que realizan frecuentes viajes a su hogar en Limón. Aquellos que se han ido y se han asimilado a la cultura dominante es posible que hayan mantenido su conocimiento del CL como segunda lengua y que ocasionalmente la usen. Aquellos que todavía ocupan su hogar tradicional en Limón, sin embargo, no la han perdido. Así es que, la concentración de hablantes del CL en la provincia de Limón, particularmente los que aún viven a lo largo de "The Line" (del antiguo tren que unía a las poblaciones del Valle de la Estrella) contribuyen a la vitalidad del grupo, por el simple hecho de que sus frecuentes interacciones verbales los ayuda a mantener sus sentimientos de solidaridad (1977: 313). Sin embargo, en la capital de la provincia de Limón, los viejos barrios de afro-costarricenses han tenido que permitir una afluencia de josefinos, y por lo tanto se han convertido en comunidades mixtas. La proporción de los miembros afro-costarricenses comparados con los que han llegado de afuera es posible que haya afectado las relaciones entre los miembros de los primeros. En realidad, como la ley en Costa Rica hasta muy recientemente ha prohibido obtener información sobre la etnicidad de los habitantes en los censos, no han habido estadísticas disponibles que puedan avalar estas observaciones.

En cuanto a los factores de los números de los grupos, como lo ha dicho Akira Yamamoto (2004: 177), el número absoluto de hablantes no siempre determina las posibilidades que el grupo pueda tener de mantener su lengua, ya que hay muchas otras variables que tienen un rol importante en el potencial de supervivencia. Tales son el índice de adquisición de los niños, la actitud respecto de la lengua de toda la comunidad, y el nivel del impacto de otras lenguas que puedan constituir una amenaza. 
El índice de nacimientos y el aumento en la proporción de matrimonios mixtos también pueden ser calculados objetivamente como "índices de retención de la lengua" (Fishman 1972: 1066). Los grupos subordinados generalmente tienen más vitalidad si su índice es alto; sin embargo, la incidencia de matrimonios mixtos de hablantes del CL-E es muy probable que resulte desfavorable para el grupo subordinado, porque generalmente son el resultado de los grupos de jóvenes que han emigrado. Han dejado atrás sus comunidades tradicionales en búsqueda de educación o mejores oportunidades ocupacionales y económicas que se encuentran en el medio hispánico de San José, la ciudad capital. Los patrones de inmigración también han sido perjudiciales para el mantenimiento del CL. Los hispanos del Valle Central que se han trasladado a Puerto Limón en busca de empleos, como resultado de las políticas neo-liberales adoptadas por el gobierno, así como los inmigrantes nicaragüenses que se escaparon de las guerras en su propio país han contribuido a aumentar los matrimonios mixtos mencionados, poniendo así en peligro el futuro colectivo del contexto interno de los grupos afro-costarricenses, especialmente en lo que respecta a su lengua.

Considerando el "factor número", el pronóstico de la manutención del CL es dudoso siendo optimista, y definitivamente contra toda posibilidad de mantener la lengua, si se es pesimista. Claro que debemos recordar que no sólo los números determinan su destino definitivo.

En cuanto a los factores de apoyo institucional, aun cuando el gobierno de Costa Rica no ha expresado apoyo formal al LC, es cierto que ha establecido la enseñanza del inglés estándar como segundo idioma en todas las escuelas públicas de la nación. Considerando que el criollo es un continuo que va del basilecto al inglés limonense estándar (en su acrolecto), este hecho puede considerarse un estímulo para los hablantes del LC, quienes pueden recalcular su valor en términos de su capacidad de dominar el inglés mucho más fácilmente que sus contrapartes hispánicos. Un apoyo informal de la comunidad puede atribuirse al grado de cohesión que lograron las minorías cuando se organizaron en el pasado como grupos de presión. Esto ocurrió a principios del siglo veinte, cuando los sindicatos organizaron huelgas contra la United Fruit Company basadas en la solidaridad obtenida por la minoría para luchar por sus propios intereses. De modo que el trabajo y el desarrollo en los sectores públicos y privados de la economía y de las instituciones educativas son determinantes del grado de vitalidad que pueden conseguir los hablantes del CL. Además, varias universidades han abierto sucursales en la provincia de Limón en el presente.

\section{Conclusiones}

Aun cuando la evaluación cuantitativa de los datos no está disponible en este momento, una interpretación cualitativa parece indicar que tanto la alternancia de códigos como las sustituciones morfológicas son un recurso básico de la comunicación entre el CL y el E, usado para marcar importantes funciones sociolingüísticas del discurso. La lengua 'prestigiosa' (E) actúa aquí como una fuerza unidireccional que contribuye al avance social del individuo. ¿Podría ser que uno interpretara la coexistencia del CL y del E por más de cien años como un indicador de que la alternancia de los códigos es un puente por el cual la experiencia bicultural de los hablantes mantiene a la lengua minoritaria intacta, por lo menos por ahora? o ¿es el impacto del E sobre el CL (como lengua Matriz) tan grande que tendrá que ser evaluado en términos del desplazamiento de la lengua y aún peor, como la muerte de la lengua en el futuro próximo?

Como Thomason (2001: 223-225) lo dice, no es tan fácil definir qué es un idioma muerto, o lo que es un hablante de una lengua, en realidad. Podríamos preguntarnos, sin 
embargo, si estos tipos de interferencias morfológicas pueden llevar eventualmente a la muerte de una lengua. Como Thomason (2001: 230) también apunta, la mayoría de los procesos lingüísticos que tienen lugar en situaciones en que una lengua muere son también comunes en situaciones en que existen lenguas en contacto, tal como es el caso ejemplificado en este estudio. Por ejemplo, los préstamos son muy comunes en situaciones de contacto, no solamente en casos de la muerte de lenguas. Sin embargo, si la gente que introduce interferencias morfológicas son hablantes fluidos de la lengua que está en 'la fila de la muerte', sus interferencias pueden ser consideradas préstamos. Si, por el contrario, son introducidos por hablantes que han aprendido la lengua en peligro de extinción como segunda lengua, entonces estas interferencias pueden ser consideradas como agentes del escuadrón de la muerte (i.e."killing squad”).

Muysken (2000: 264) considera que se puede distinguir entre préstamos, que él llama 'convergentes' (el resultado de influencias bidireccionales) y aquéllos que él llama 'préstamos gramaticales.' Presenta la posibilidad de sincronizar los modelos analíticos que él y Thomason desarrollaron, tomando en cuenta la multidimensionalidad de las diferentes estrategias en el uso del bilingüismo. En todo caso, Muysken concluye que la mezcla de códigos extiende el repertorio de los hablantes bilingües, pero que pagan un cierto costo por esa expansión. Uno podría preguntarse si el desplazamiento de una lengua es uno de esos costos.

Quizás, después de todas estas consideraciones, es totalmente posible que si los hablantes del CL valoran su identidad -especialmente enfrentando las presiones socioeconómicas del presente hacia la universalización de la cultura- podría bien ser que resulte en un reconocimiento de la importancia de su lengua como una expresión de su dignidad y autoestima.

\section{Notas}

1. Algunos de los ejemplos de alternancia de códigos mencionados y los resultados de aplicar el modelo de Myers-Scotton sobre alternancia de códigos han sido publicados anteriormente en español en un artículo titulado "Mecanismos de cambios lingüísticos inducidos por contacto," en Dinámica lingüística de las lenguas en contacto, editado por Claudine Chamoreau and Yolanda Lastra, Hermosillo, 2005, pp. 331355. Todos los ejemplos que ilustran substituciones e integraciones son presentados aquí por primera vez, así como la evaluación de la vitalidad de la lengua.

2. De acuerdo con un estudio de marcadores genéticos que el Dr. Ramiro Barrantes (1992) condujo en la Universidad de Costa Rica, el autor señaló los siguientes porcentajes para la composición de la población de Costa Rica: el elemento caucásico varía entre un 40 a $60 \%$; el componente negroide va desde un 10 a 40\%, y el amerindio entre un 20 a 30\% (citado en Bozzoli 1993:2).

3. Las lenguas indígenas más comúnmente habladas en Costa Rica son las siguientes: Bribri, Cabécar, Maleku o Guatuso, Térraba y Guaymí.

4. Un consultor a quien yo entrevisté en Limón hace unos diez años, me dijo que como trabajaba en un oficina hablando español todo el día mientras que su esposa hacía lo mismo, cuando ambos regresaban a su hogar a la noche, cansados y listos para pasar tiempo con sus niños, el hablar CL con ellos implicaba un esfuerzo extra que no tenían ánimo de emprender. Por eso ellos educaban a sus niños prácticamente sólo en español.

5. Un estudio cuantitativo posterior al presente demuestra que la vitalidad del criollo limonense es tal que no parece estar en peligro de desaparecer por el momento. 


\section{Bibliografía}

Arends, Jacques; Pieter Muysken y Smith Norval (Eds.). 1995. Pidgins and Creoles: an Introduction. Amsterdam: John Benjamins Publishing Company.

Bozzoli, María Eugenia. 1995-1996. “La población costarricense: diversidad, tolerancia y discriminación”. Revista Herencia. 7-8 (1-2): 131-148.

Chamoreau, Claudine y Yolanda Lastra (Eds.). 2005. Dinámica lingüística de las lenguas en contacto. Hermosillo: Universidad de Sonora.

Dorian, Nancy (Ed.). 1989. Investigating Obsolescence. Studies in Language Contraction and Death. Cambridge: Cambridge University Press.

Finegan, Edward y John Rickford (Eds.). 2004. Language in the U.S.A.: Themes for the Twenty-first Century. Cambridge: Cambridge University Press.

Fishman, Joshua. 1972. Language and Nationalism. Massachusetts: Newbury House.

Foley, William. 1998. Anthropological Linguistics: An Introduction. Massachusetts: Blackwell.

Giles, Howard (Ed.). 1977. Language, Ethnicity and Intergroup Relations. London: Academic Press.

Giles, Howard; Richard Bourhis y Donald Taylor. 1977. "Towards a Theory of Language in Ethnic Group Relations”. En: Giles (Ed.), 307-344.

Gumperz, John. 1982. “Conversational code-switching”. Discourse Strategies. Cambridge: Cambridge University Press.

Haugen, Einar. 1956. Bilingualism in the Americas: A Bibliography and Research Guide. Alabama: University of Alabama Press.

Herzfeld, Anita. 2002. /mekaytelyuw/: la lengua criolla (de Costa Rica). San José: Editorial de la Universidad de Costa Rica.

2005. "Mecanismos de cambios lingüísticos inducidos por contacto". En: Chamoreau y Lastra (Eds.), 333-355.

Holm, John (Ed.). 1983. Central American English. Heidelberg: Groos.

Jacobson, Rodolfo (Ed.). 1998. Trends in Linguistics. Studies and Monographs 106. Codeswitching Worldwide. Berlin: Mouton de Gruyter. 
Kindell, Gloria y M. Paul Lewis (Eds.). 2000. Assessing Ethnolinguisitc Vitality: Theory and Practice. Dallas: SIL International.

Miller, Carolyn. 2000. "Modifying Language Beliefs: a Role for Mother-Tongue Advocates?". En: Kindell y Lewis (Eds.), 167-187.

Myers- Scotton, Carol. 1993a. Social motivation for code-switching: evidence from Africa. Oxford: Clarendon Press.

1993b. Dueling languages: Grammatical structure and code-switching. Oxford: Oxford University Press.

Muysken, Pieter. 2000. Bilingual Speech: A Typology of Code-Mixing. Cambridge: Cambridge University Press.

Rindler, Rosita. 1998. "Code-switching as an indicator for language shift? Evidence from Sardinian-Italian bilingualism”. En: Jacobson (Ed.), 221-248.

Smith, Norval. 1995. "An annotated list of creoles, pidgins, and mixed languages". En: Arends, Muysken y Smith (Eds.), 331-374.

Spence, Marva. 1993. A Case Study of Language Shift in Progress in Port Limon, Costa Rica. Ph. D. dissertation: Georgetown University.

Thomason, Sara. 2001. Language Contact: an Introduction. Washington, D.C.: Georgetown University Press.

Weinreich, Uriel. 1953. Languages in Contact: Findings and Problems. New York: Linguistic Circle of New York.

Yamamoto, Akira y Ofelia Zepeda. 2004. "Native American Languages”. En: Finegan y J. Rickford (Eds.), 153-181. 


\section{APÉNDICE 1}

La distribución de los Criollos Ingleses de Centroamérica Tomado de Holm (1983: 8)

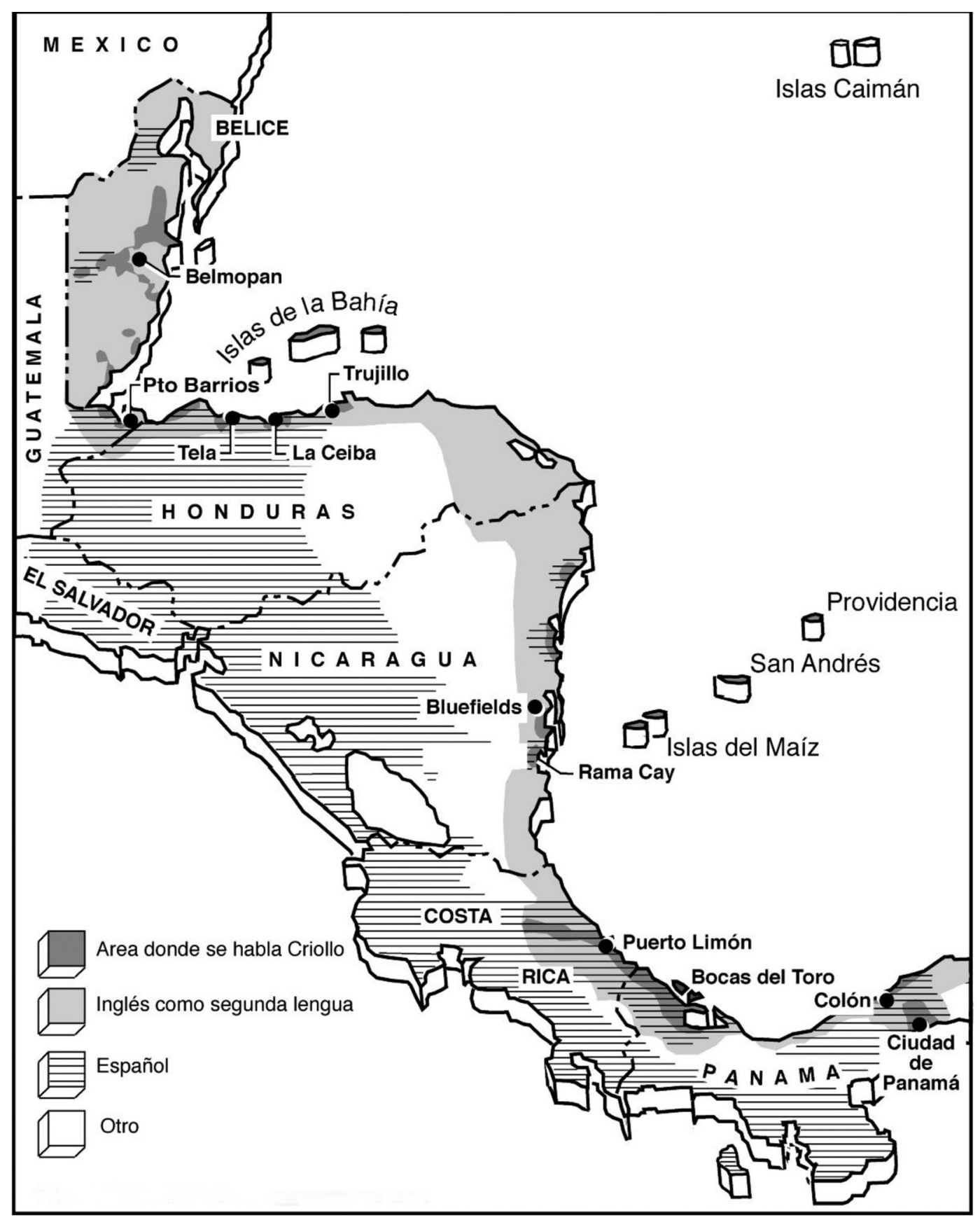




\section{APÉNDICE 2 \\ Factores que determinan la vitalidad de lenguas en contacto Adaptado de Miller (2000:169). (La traducción es mía)}

\section{Estatus y factores de desarrollo}

\subsection{Factores Económicos}

Si los hablantes de una lengua minoritaria tienen el mismo estatus económico que los hablantes de la lengua de la mayoría, es más probable que su lengua será mantenida.

\subsection{Estatus Social}

Si los hablantes de la lengua minoritaria han obtenido un nivel de aceptación social igual a la de los miembros de la lengua mayoritaria, es mucho más factible que su lengua será mantenida.

\subsection{Prestigio Socio-histórico}

Si una lengua tiene importancia religiosa o clásica, es más probable que será mantenida.

\subsection{El estatus lingüístico dentro y fuera de la comunidad}

Los idiomas que gozan de un estatus alto es más probable que se mantengan que aquéllos de más bajo estatus.

\subsubsection{Evaluación Externa}

Si los hablantes de la lengua mayoritaria evalúan a esa lengua con respeto, es muy probable que esa lengua se mantenga.

\subsubsection{Evaluación Interna}

Si los hablantes de la lengua la consideran de valor importante en su cultura, es más probable que será mantenida.

\subsubsection{El desarrollo de la lengua}

Si la lengua tiene una forma escrita y un estándar reconocido, es más posible que la lengua será mantenida.

\section{Factores Demográficos}

\subsection{Número de miembros}

Aunque es difícil de calcular (por la inseguridad de los censos), si el número de hablantes es significativo, es más probable que la lengua será mantenida.

\subsection{Distribución y concentración de hablantes}

Los grupos cuyos miembros forman una sociedad cohesiva en su arreglo espacial es más probable que mantengan su lengua. 


\subsection{Naturaleza de la situación de contacto}

Un grupo super-ordinario es más probable que mantenga su lengua que uno subordinado, y un grupo subordinado que es nativo del área (con instituciones establecidas) tiene más chances de mantener su lengua que aquéllos que han emigrado del área. Y aquéllos que se han convertido en subordinados, por colonización [(i. e. por haber sido colonizados)] o porque han sido anexados no abandonarán su lengua por otra tan fácilmente como aquéllos que han emigrado.

\section{4. Índice de nacimientos}

\subsection{Modelos de inmigración y migración}

Los grupos que no tienen movimientos migratorios significativos es más probable que mantengan su idioma.

\subsection{Modelos matrimoniales}

Los grupos que practican la endogamia (particularmente en base a matrimonios arreglados) es más probable que mantengan su lengua que aquéllos que practican la exogamia.

\section{Factores de apoyo institucional y factores de control}

\subsection{El apoyo formal $\mathrm{o}$ informal recibido de las instituciones gubernamentales}

Si la política lingüística de un gobierno es pluralista, en lugar de ser asimilacionista, es más probable que la lengua sea mantenida. Si la comunidad de habla vernácula está de acuerdo con la política lingüística del gobierno, ésta será más efectiva.

\subsection{Los factores que intervienen en el uso de la lengua}

Los que aparecen más abajo favorecen la manutención de la lengua.

3.2.1. Apoyo del hogar

Si la lengua se habla en el hogar

\subsubsection{Apoyo de la escuela}

Si se enseña la lengua en la escuela

3.2.3. Apoyo de la iglesia

$\mathrm{Si}$ se usa en funciones religiosas

3.2.4. Apoyo del comercio

Si se usa en transacciones comerciales.

3.2.5. Si la lengua se usa en los medios de comunicación

3.2.6. Si la lengua se usa en las oficinas del gobierno y en lugares donde se proveen servicios.

3.2.7. Si la lengua se usa en actividades políticas (campañas políticas, discursos)

3.2.8. Si el grupo mantiene control sobre sus actividades culturales y las conduce en su lengua 
